\title{
Ultralow-loss domain wall motion driven by a magnetocrystalline anisotropy gradient in an antiferromagnetic nanowire
}

\author{
D. L. Wen,,${ }^{1, *}$ Z. Y. Chen, ${ }^{1, *}$ W. H. Li ${ }^{1}$ M. H. Qin $\odot,{ }^{1,}{ }^{\dagger}$ D. Y. Chen, ${ }^{1}$ Z. Fan, ${ }^{1}$ M. Zeng, ${ }^{1}$ X. B. Lu, ${ }^{1}$ X. S. Gao, ${ }^{1}$ and J.-M. Liu ${ }^{1,2}$ \\ ${ }^{1}$ Guangdong Provincial Key Laboratory of Quantum Engineering and Quantum Materials and Institute for Advanced Materials, \\ South China Academy of Advanced Optoelectronics, South China Normal University, Guangzhou 510006, China \\ ${ }^{2}$ Laboratory of Solid State Microstructures, Nanjing University, Nanjing 210093, China
}

(Received 9 September 2019; revised manuscript received 13 November 2019; accepted 14 January 2020; published 18 February 2020)

\begin{abstract}
Searching for a new scheme to control the antiferromagnetic (AFM) domain wall is one of the most important issues for AFM spintronic devices. In this work, we study theoretically the domain wall motion of an AFM nanowire, driven by the axial anisotropy gradient generated by an external electric field and an electrocontrol of AFM domain wall motion in the merit of ultralow energy loss is demonstrated. The domain wall velocity depending on the anisotropy gradient magnitude and intrinsic material properties is simulated based on the Landau-Lifshitz-Gilbert equation and also deduced using the energy dissipation theorem. It is shown that the domain wall moves at a nearly constant speed for the small anisotropy gradient, and this motion is accelerated for the large gradient due to the enlarged domain wall width. While the domain wall mobility is independent of the lattice dimension and types of the domain wall, it can be enhanced by the Dzyaloshinskii-Moriya interaction. In addition, the physical mechanism for much faster AFM wall dynamics than ferromagnetic wall dynamics is qualitatively explained. This work unveils a promising strategy for controlling the AFM domain walls, benefiting the future of AFM spintronic applications.
\end{abstract}

DOI: 10.1103/PhysRevResearch.2.013166

\section{INTRODUCTION}

Nowadays, the interest in antiferromagnets increases significantly due to the promising application potentials of the so-called antiferromagnetic (AFM) spintronics [1,2]. Comparing with ferromagnet based storage devices, AFM spintronic devices are more stable against magnetic field perturbations and could be designed with high element densities without producing a stray field, attributing to zero net magnetization and the ultralow susceptibility of AFM elements [3-6]. Moreover, AFM materials show fast magnetic dynamics [7], including ultrahigh frequency spin wave modes resulting from the complex spin configurations, and are highly favored for future devices [8].

One of the major challenges for these applications is how to control an AFM domain wall. A number of schemes for driving the AFM domain wall motion, including spin waves [9], spin-orbit torques [10,11], asymmetric magnetic fields [12], and thermal gradients [13-15], have been proposed. For example, a wall motion speed as high as $\sim 30 \mathrm{~km} / \mathrm{s}$, driven by electric current induced Néel spin-orbit torques, was predicted

\footnotetext{
*These authors contributed equally to this work.

†qinmh@scnu.edu.cn

Published by the American Physical Society under the terms of the Creative Commons Attribution 4.0 International license. Further distribution of this work must maintain attribution to the author(s) and the published article's title, journal citation, and DOI.
}

in CuMnAs [16] and $\mathrm{Mn}_{2} \mathrm{Au}$ [17]. This speed is nearly two orders of magnitude larger than that for ferromagnetic (FM) domain wall motion typically, although electric current control scheme will have high energy cost. Furthermore, it was revealed that the competition between entropic torque and Brownian force under a temperature gradient determines the wall motion direction. Importantly, in these control schemes, the AFM wall motion remains nontilted and high wall mobility is expected since no walker breakdown $[18,19]$, which limits the motion of a FM domain wall, is available in the AFM wall motion.

While these proposed schemes are meaningful for future experiments and device design, several shortcomings can be found and they may be detrimental in some cases. For instance, the Néel spin-orbit torque is available only in some specific antiferromagnets with locally broken inversion symmetry $[10,11]$. This torque, however, drives the neighboring domain walls to approach and then annihilate with each other, seriously hindering its applications. Some schemes, such as the temperature gradient and spin wave driven wall motion $[9,14]$, theoretically sound great but are difficult to implement. It is also noted that some of these schemes rely essentially on the electric current driven mechanism [10,12], which is highly deficient for its high energy loss due to the Joule heating. So, the search is still on the way to find an alternative scheme that is well-controlled, energy-saving, and high efficient for future AFM spintronic applications.

Electric field rather than electric current control would be highly preferred if one is concerned with the energy cost, which is detrimental for ultradensity memories. Indeed, 
electric field control, or electrocontrol, of magnetism has been an issue deserving full commitment. Fortunately, electric field controlled magnetic anisotropy has been experimentally revealed in magnetic heterostructures [20-23], and the anisotropy gradient can be obtained through an elaborate structure design such as wedged heterostructures [24]. Under such a gradient, the AFM domain wall tends to move towards the low anisotropy side in order to reduce the free energy. As a matter of fact, the anisotropy gradient has been proven to efficiently drive the skyrmion motion [25-27], and this scheme should be also utilized to control the AFM domain wall motion with preferred merit. However, so far, no report on the dynamics of AFM domain walls under such anisotropy gradient is available, and this subject urgently deserves to be investigated theoretically as the first step towards promoting the application process for AFM spintronics.

In this work, we study the anisotropy gradient driven AFM domain wall motion in a nanowire with the AFM domain structure. The dynamics and its dependence on the gradient magnitude and intrinsic physical parameters will be simulated based on the Landau-Lifshitz-Gilbert (LLG) equation and also calculated based on the energy dissipation theorem. It is predicted that the domain wall does shift at a nearly constant speed when the anisotropy gradient is small and the wall motion would be accelerated if the anisotropy gradient increases because of the widened domain wall during the motion stage. It is confirmed that the observed phenomena are independent of the lattice dimension and wall type. Moreover, the role of Dzyaloshinskii-Moriya (DM) interaction is discussed specifically and its influence on the spin dynamics is addressed, benefiting a lot future material design and dynamic manipulation. In addition, the physical mechanism of the AFM wall dynamics being faster than FM wall dynamics is also qualitatively explained.

\section{MODEL AND COMPUTATIONAL DETAILS}

Without losing generality, we study a one-dimensional AFM model with an isotropic Heisenberg exchange between the nearest neighbors and a uniaxial anisotropy term [28]:

$$
H=J \sum_{\langle i, j\rangle} S_{i} \cdot S_{j}-\sum_{i} K_{i}^{z}\left(S_{i}^{z}\right)^{2},
$$

where $J>0$ is the AFM coupling constant, $\boldsymbol{S}_{i}=\boldsymbol{\mu}_{i} / \mu_{s}$ represents the normalized magnetic moment at site $i$ with three components $\left(S_{i}^{x}, S_{i}^{y}, S_{i}^{z}\right)$. The second term in Eq. (1) is the anisotropy energy with the easy axis along the $z$ direction (i.e., nanowire axis), and the uniaxial anisotropy constant $K_{i}^{z}$ at site $i$ being described by $K_{i}^{z}=K_{0}+i a \times \Delta K$, where $K_{0}$ is the anisotropy constant at the low anisotropy end and $\Delta K$ describes the anisotropy gradient magnitude, $a$ is the lattice constant.

The AFM dynamics is investigated by solving the LLG equation based on the atomistic spin model [29,30]:

$$
\frac{\partial S_{i}}{\partial t}=-\frac{\gamma}{\mu_{s}(1+\alpha)^{2}} S_{i} \times\left[H_{i}+\alpha\left(S_{i} \times H_{i}\right)\right],
$$

where $\gamma$ is the gyromagnetic ratio, the Gilbert damping constant $\alpha=0.002$ is chosen referring to realistic materials such as $\mathrm{NiO}$ [31], $H_{i}=-\partial H / \partial S_{i}$ is the effective field

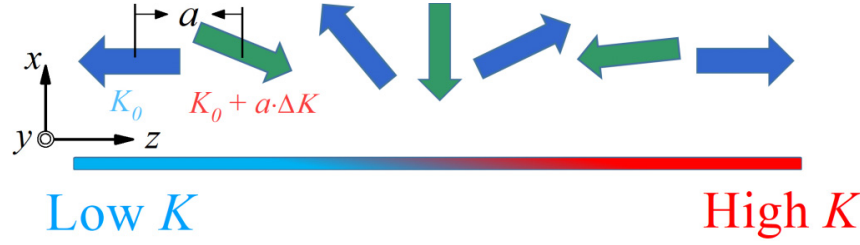

FIG. 1. Illustration of an AFM domain wall in a nanowire under an anisotropy gradient, where low $K$ and high $K$ refer to the anisotropy magnitude.

including the exchange field $H_{i}^{\text {ex }}=J \sum S_{j}$ and the anisotropy field $H_{i}^{\text {an }}=K_{0} S_{i}+i a \Delta K S_{i}$. Unless stated elsewhere, the LLG simulations are performed on the nanowire lattice with $1 \times 1 \times 400$ spins with open boundary conditions using the fourth-order Runge-Kutta method with a time step $\Delta t=$ $1.0 \times 10^{-4} \mu_{s} / \gamma J$. The local staggered magnetization $2 \mathbf{n}=$ $\mathbf{m}_{1}-\mathbf{m}_{2}$ is calculated to describe the spin dynamics, where $\mathbf{m}_{1}$ and $\mathbf{m}_{2}$ are the magnetizations of the two sublattices. After sufficient relaxation of the domain structure, the anisotropy gradient is applied to drive the domain wall motion, as schematically depicted in Fig. 1.

\section{RESULTS AND DISCUSSION}

\section{A. Domain wall motion}

We first demonstrate the domain wall motion upon a finite anisotropy gradient $\Delta K$. It is clear to see no identifiable wall motion at $\Delta K=0$. The motion trajectory at $K_{0}=$ $0.01 \mathrm{~J}$ and $\Delta K=6 \times 10^{6} \mathrm{~J} / a$, at five given times, is presented in Fig. 2(a), where the domain wall profiles are plotted. The domain wall motion in roughly steady state is clearly
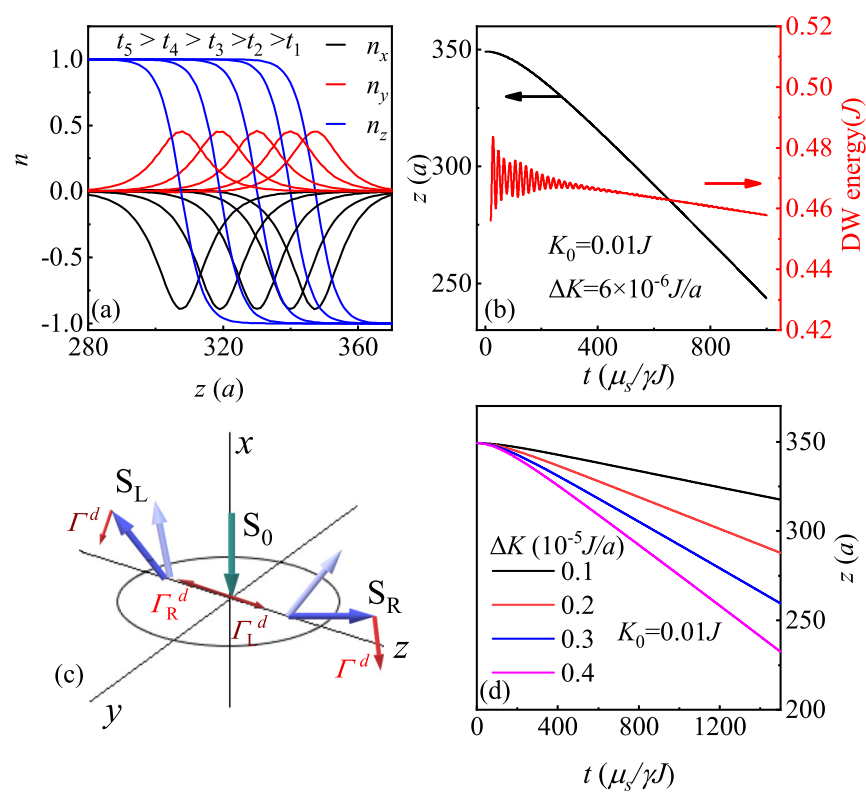

FIG. 2. (a) Spatial profile of the domain wall at different times, and (b) the domain wall position and energy as functions of time for $\Delta K=6 \times 10^{-6} \mathrm{~J} / a$ and $K_{0}=0.01 \mathrm{~J}$. (c) A schematic depiction of torques acting on domain wall spins under the anisotropy gradient, and (d) the position of domain wall vs time for various $\Delta K$ at $K_{0}=0.01 J$. 
demonstrated as long as a finite anisotropy gradient is imposed. The wall always shifts from the high anisotropy side to the low anisotropy side, with a nearly constant speed. The simulated wall position $(z)$ and wall energy $\left(E_{\mathrm{DW}}\right.$, the internal energy difference between the systems with and without the wall) as functions of time $t$ are shown in Fig. 2(b). It is seen that the wall position $z$ decreases very slowly in the very beginning and then the wall motion is accelerated up to a state with roughly constant speed. The wall energy $E_{\mathrm{DW}}$, however, experiences serious fluctuations in the first period, and then reaches a state where $E_{\mathrm{DW}}$ approximately linearly decreases with time $t$ and exhibits very weak fluctuations.

It is clearly demonstrated that the domain wall motion towards the low anisotropy side is a spontaneous process. In the first period, the wall symmetry is broken slightly by introducing an anisotropy gradient, resulting in the distortion of wall structure and serious fluctuation of $E_{\mathrm{DW}}$. Moreover, the trajectory of $\mathbf{n}$ depends not only on its instantaneous position but also on the spin dynamics [32]. Specifically, $\mathbf{n}$ tends to continue its path even after reaching the equilibrium position (along its effective field), accounting for the weak fluctuations of $E_{\mathrm{Dw}}$ after the wall structure is readjusted.

As a matter of fact, the wall motion can be qualitatively understood from the competition among various torques acting on the wall spins, as depicted in Fig. 2(c). In the absence of anisotropy gradient, the domain wall configuration is symmetric with respect to its central plane. This symmetry is broken if one considers a nonzero anisotropy gradient. Specifically, for the two spins $\left(S_{\mathrm{L}}\right.$ and $S_{\mathrm{R}}$ ) neighboring the spin $S_{0}$ exactly on the wall central plane, the damping torque on $S_{\mathrm{L}}$, resulting from the anisotropy term, is smaller than that on $S_{\mathrm{R}}$, leading to the fact that $S_{\mathrm{L}}$ deviates more from the easy axis than $S_{\mathrm{R}}$ does. As a result, the damping torque $\left(\boldsymbol{\Gamma}_{R}^{d}\right)$ on $S_{0}$ from the exchange interaction with $S_{\mathrm{R}}$ is larger than the damping torque $\left(\boldsymbol{\Gamma}_{L}^{d}\right)$ from $S_{\mathrm{L}}$, resulting in the net damping torque $\sim\left(\boldsymbol{\Gamma}_{\mathrm{R}}^{d}+\boldsymbol{\Gamma}_{\mathrm{L}}^{d}\right)$ which efficiently drives the wall motion toward the low anisotropy region. Undoubtedly, the net driving torque increases with increasing $\Delta K$, which significantly enhances the wall motion speed, as clearly shown in Fig. 2(d) where the wall position $z$ is plotted as a function of time $t$ at several $\Delta K$.

In order to uncover the underlying physics more clearly, we calculate the wall motion speed from the perspective of energy conservation. Considering the wall motion as a consequence of wall energy dissipation, one notes that the wall energy $E_{\mathrm{DW}}=2\left(2 J K_{c}\right)^{1 / 2}$, where $K_{c}$ is the anisotropy constant on spin $S_{0}$ [33], noting $K_{c}>K_{0}$ if $\Delta K>0$. The Rayleigh dissipation function $R=a \rho \int \dot{\mathbf{n}}^{2} d z / 2$ is introduced to describe the energy dissipation of the whole lattice [34], where $\rho=$ $\mu_{s} / \gamma a$ is the density of staggered spin angular momentum per unit cell and $\dot{n}$ represents the derivative with respect to time. Subsequently, according to the classical mechanics, we obtain

$$
d E_{\mathrm{DW}} / d t=-2 R \text {. }
$$

A necessary mathematical substitution leads to the wall velocity $v$ (see Appendix for details):

$$
v=-\frac{\gamma \lambda^{2} \Delta K}{\alpha \mu_{s}}, \quad \lambda=a\left(\frac{J}{2 K_{c}}\right)^{1 / 2},
$$

where $\lambda$ is the domain wall width.
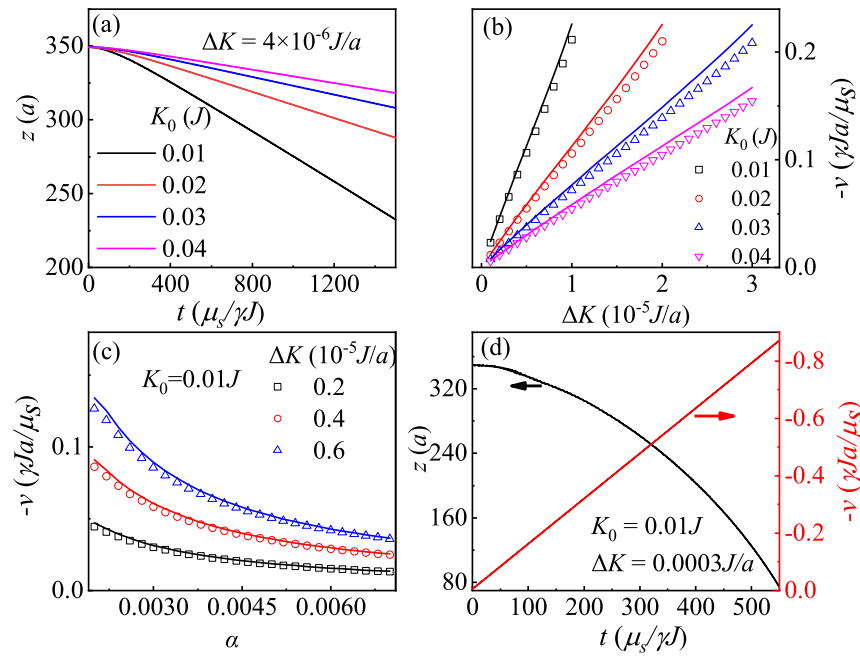

FIG. 3. (a) The position of the domain wall as a function of time for various $K_{0}$ at $\Delta K=4 \times 10^{-6} \mathrm{~J} / a$. The simulated (empty points) and calculated (solid lines) velocities as functions of (b) $\Delta K$ for various $K_{0}$ and $\alpha=0.002$, and (c) $\alpha$ for various $\Delta K$ at $K_{0}=0.01 J$. (d) The simulated domain wall position and velocity as functions of time for large $\Delta K=0.0003 \mathrm{~J} / \mathrm{a}$.

Equation (4) suggests that the wall velocity depends not only on the anisotropy gradient $\Delta K$ but also on the anisotropy constant $K_{c}$. It implies that a larger anisotropy $\Delta K$ makes the wall motion faster but a larger $K_{c}$ does the opposite. This phenomenon can be understood from two aspects. On one hand, the magnitude of the anisotropy gradient determines the strength of the driving torque on the domain wall. A larger gradient enhances the driving torque so that the domain wall moves at a higher speed. On the other hand, the domain wall width $\lambda$, depending on the anisotropy constant, significantly affects the wall motion, as shown in Eq. (4), i.e., $v \sim \lambda^{2}$. Thus the wall width is decreased with the increasing anisotropy constant, reducing the domain wall motion. This result is confirmed from our simulations. Figure 3(a) presents the simulated wall position $z$ as a function of $t$ for various $K_{0}$ at $\Delta K=4 \times 10^{-6} \mathrm{~J} / a$, noting that a large $K_{0}$ also implies a large $K_{c}$. Certainly, an enhanced anisotropy is expected to suppress the domain wall motion seriously, as shown in Figs. 3(a) and 3(b). In Fig. 3(b), the simulated speeds (empty dots) and calculated ones (solid lines) from Eq. (4) are plotted together for a comparison. The good consistence between the simulation and model is demonstrated on one hand, and on the other hand it is revealed that the anisotropy magnitude $\left(K_{0}\right.$ or $K_{c}$ ) and anisotropy gradient $(\Delta K)$ play opposite roles in controlling the domain wall motion, enabling an accelerated and decelerated wall motion, respectively.

The effect of the damping constant $\alpha$ on the wall motion is also investigated, and the corresponding results are shown in Fig. 3(c), which gives the simulated and calculated velocities as functions of $\alpha$ for various $\Delta K$ at $K_{0}=0.01 \mathrm{~J}$. It is noted that an enhanced damping term always lowers the wall mobility, and the velocity decreases with increasing $\alpha$. Furthermore, the wall width is simply considered to be a constant during the wall motion, which well describes the cases of small $\Delta K$. However, for large $\Delta K$, the wall width is expected to 

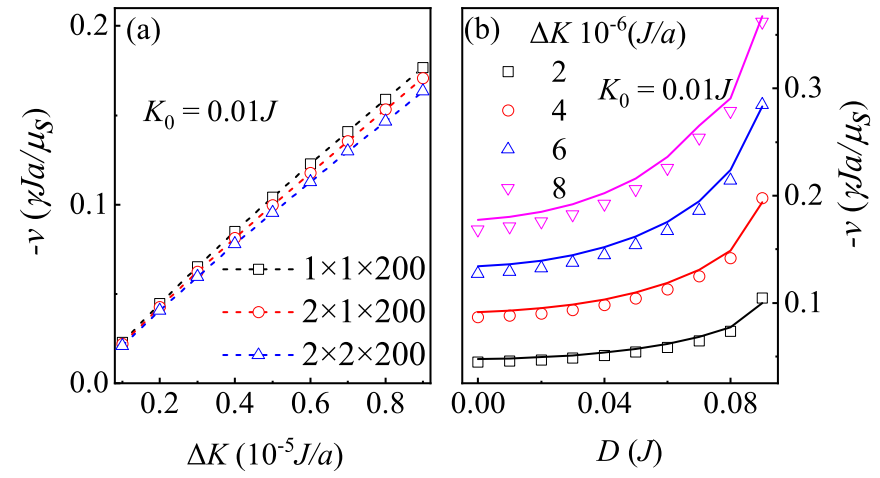

FIG. 4. (a) The simulated domain wall velocity as a function of $\Delta K$ for various lattice sizes, and (b) the simulated (empty points) and calculated (solid lines) velocities as functions of $D$ for various $\Delta K$.

increase with the wall motion toward the weak anisotropy side, resulting in an additional acceleration of the wall motion. In order to better understand the acceleration behavior, we investigate the wall motion under huge anisotropy gradient $\Delta K=0.0003 \mathrm{~J} / a$. The simulated wall position and velocity are presented in Fig. 3(d), which clearly shows that the velocity linearly increases with $t$.

Furthermore, a $90^{\circ}$ AFM domain wall could be developed in real materials such as CuMnAs due to the current polarity and may play an essential role in future applications [35]. Here, the dynamics of the $90^{\circ} \mathrm{AFM}$ wall is also investigated by introducing the cubic anisotropy $K\left(S_{x}^{2} S_{y}^{2}+S_{y}^{2} S_{z}^{2}+S_{z}^{2} S_{x}^{2}\right)$ and additional hard axis anisotropy $K_{y} S_{y}^{2}$ in the model [36]. In this case, the wall energy goes to $E_{\mathrm{DW}}=\left(J K_{c} / 2\right)^{1 / 2}$, exactly a quarter of the energy of the $180^{\circ}$ wall under the same parameters. However, the Rayleigh dissipation function $R$ of the $90^{\circ}$ wall is also one-fourth that of the $180^{\circ}$ wall, as explained in the appendix, resulting in the same velocity. As a matter of fact, this behavior has also been confirmed by the LLG simulations, and the corresponding results are not shown here for brevity.

\section{B. Lattice dimension and DM interaction}

So far, the anisotropy gradient driven AFM wall motion has been studied based on a one-dimensional model. However, the results and conclusions also apply to magnetic films and bulks, as shown in Fig. 4(a), which presents the simulated wall velocity as a function of $\Delta K$ for various lattice dimensions at $K_{0}=0.01 J$ and $\alpha=0.002$, where $J$ is the in-plane exchange interaction. All the curves well coincide with each other especially for small $\Delta K$, indicating the independence of the wall velocity on the lattice dimension. This phenomenon can be qualitatively understood from the energy landscape. At small $\Delta K$, the wall structure is almost the same as the static wall. In this case, the neighboring spins in every cross-section perpendicular to the $z$ axis still align antiparallel to each other, and the exchange energy is unchanged. Thus the energy difference between neighboring unit cross sections only arises from the single-ion anisotropy term, which is hardly affected by the lattice dimensions, resulting in the invariant force density exerted on the wall.
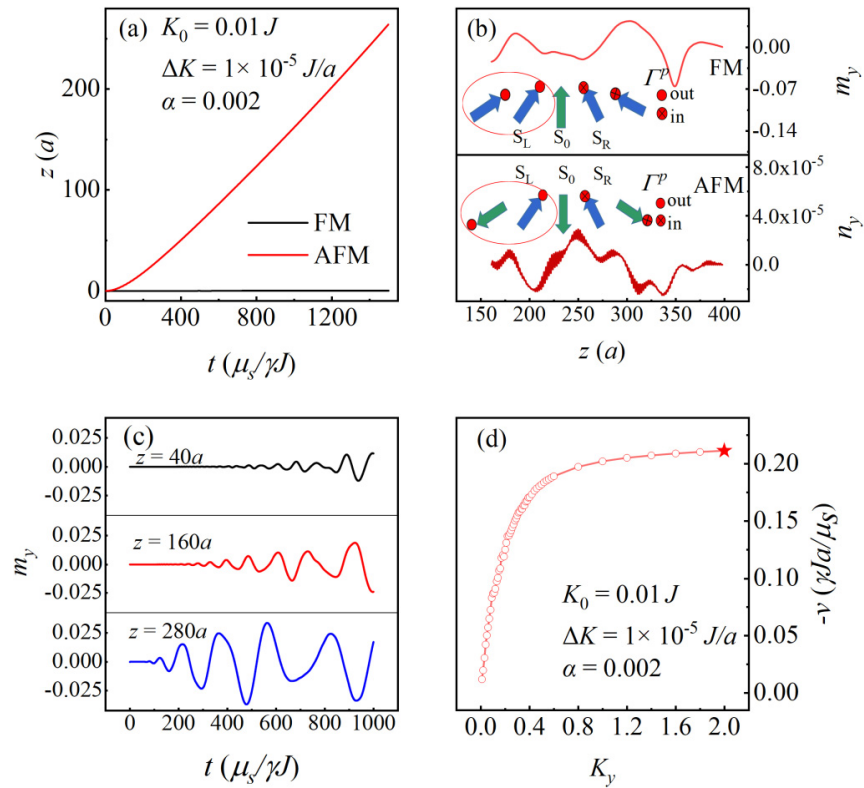

FIG. 5. (a) The displacements of the domain walls of the AFM and FM systems as functions of time. (b) The local $m_{y}$ (top) of the FM system and $n_{y}$ (bottom) of the AFM system, the inserts are the schematic depictions of the precession torques. (c) The evolution of $m_{y}$ at various positions, the domain wall center is located at $z=350 a$. (d) The simulated FM wall velocity as a function of $K_{y}$.

Moreover, we check the dependence of the domain wall dynamics on the DM interaction, which may be available in some realistic materials [37,38]. Here, the DM interaction energy $\Sigma_{i} \mathbf{D} \cdot\left(\mathbf{S}_{i} \times \mathbf{S}_{i+1}\right)$ with $\mathbf{D}=D(0,0,1)$ is introduced in the one-dimensional model, and the LLG simulated results are presented in Fig. 4(b), where the $v(D)$ curves upon various $\Delta K$ are plotted. For a fixed $\Delta K, v$ gradually increases with increasing $D$, indicating that the DM interaction enhances the wall motion, similar to the earlier report $[39,40]$. When the DM interaction is considered, the domain wall energy decreases to $E_{\mathrm{DW}}=2\left(2 J K_{c}-D^{2}\right)^{1 / 2}[9,41]$, resulting in the increase of the wall width and the enhancement of the wall mobility. In detail, the wall width is increased up to [42]

$$
\lambda=\lambda_{0}\left(1-D^{2} / 2 J K_{c}\right)^{-1 / 2},
$$

with $\lambda_{0}=a\left(J / 2 K_{c}\right)^{1 / 2}$.

In Fig. 4(b), the calculated velocity curves are also plotted with solid lines, which are well consistent with the simulated results.

\section{Antiferromagnet beats ferromagnet}

In this section, we discuss the physical mechanism for the ultrafast dynamics of an AFM wall under the anisotropy gradient through a detailed comparison with the FM domain wall. Comparing with the AFM wall, the FM wall can hardly be driven by the anisotropy gradient, as clearly shown in Fig. 5(a) where the simulated wall displacements of the AFM system and FM system with the exchange interaction $-J$ for $\Delta K / K_{0}=1 \times 10^{-3} a^{-1}$ and $\alpha=0.002$ are presented. For a FM wall, the precession torques on $\mathbf{S}_{R}$ and $\mathbf{S}_{\mathrm{L}}$ from the gradient are antiparallel and compete with each other, 
resulting in a strong spin fluctuation, as shown in the top half of Fig. 5(b) where the $y$ component of local spin $m_{y}$ is presented. Moreover, the spin fluctuation cannot be suppressed by the FM interaction but propagates along the nanowire from the domain wall center, as confirmed in Fig. 5(c) where the evolutions of $m_{y}$ at $z=40 a, 160 a$, and $280 a$ are plotted, noting that the domain wall center is located at $z=350 a$. As a result, the excitation and propagation of spin fluctuation act as dominating energy dissipations since the precession torque is much larger than the damping torque, resulting in the very low driving efficiency of the FM wall.

On the other hand, for the AFM system, the high mobility of the AFM wall is obtained, due to the fact that the spin fluctuation is significantly suppressed by the AFM interaction, as shown in the bottom half of Fig. 5(b) where we present the $y$ component of the staggered magnetization $n_{y}$ of the system and depict the precession torque acting on the AFM spins. Thus, this work unveils another origin for ultrafast dynamics of an AFM system. However, the weak spin fluctuation also slightly speeds down the motion of the AFM domain wall, resulting in a noticeable discrepancy between the simulations and analytical calculations especially for large $\Delta K$, which significantly enhances the spin fluctuation, as shown in Fig. 3.

In addition, a high mobility of the FM wall is expected and also confirmed in our simulations when the spin fluctuation is suppressed by introducing additional hard-axis anisotropy $K_{y} S_{y}^{2}$ in the model. Figure 5(d) shows the simulated FM wall velocity as a function of $K_{y}$. With the increase of $K_{y}$, the spin fluctuation is gradually suppressed, and the velocity increases to a saturation value approximating that of the AFM wall.

\section{Brief discussion}

While we are pursuing the faster, the smaller, the denser for spintronic devices, the domain wall motion efficiently driven by low energy consumption is always a core concern for spintronics. Thus, finding new energy-saving methods of controlling the domain walls in an antiferromagnet, regardless of it being metal or insulator, is still of great importance for future applications. In this work, the anisotropy gradient has been clearly revealed to efficiently drive the AFM domain wall motion.

Moreover, it is noted that defects are inevitable in real materials and affect the magnetic dynamics. In one of our earlier works, the depinning of an AFM domain wall in notched nanostructures driven by the Néel field was theoretically investigated [7]. It has been demonstrated that the depinning field depends markedly on the damping constant. More interestingly, the work also unveils the difference in the depinning mechanisms of an antiferromagnetic domain wall and a ferromagnetic domain wall, which results in a depinning speed typically two orders faster than the latter. To some extent, similar impact of potential defects is expected for the anisotropy gradient driven domain wall motion.

In order to help one to catch up the results easily, we estimate the real value of the domain wall velocity taking $\mathrm{NiO}$ as an example [43]. Specifically, for $\Delta K / K_{c}=1 \times 10^{-4} a^{-1}$ and $\alpha=0.002$, the speed of the wall for $\mathrm{NiO}$ with the exchange stiffness $A \approx 5 \times 10^{-13} \mathrm{~J} / \mathrm{m}, a \approx 4.2 \AA, \mu_{s} \approx 1.7 \mu_{B}\left(\mu_{B}\right.$ is the Bohr magneton) is estimated to be $\sim 100 \mathrm{~m} / \mathrm{s}$. More im- portantly, the proposed modulating method is expected to be more energy saving and producing less Joule heat compared to those electric current driving methods. Thus our work unveils a promising method of controlling AFM walls, and does provide useful information for future spintronic applications.

\section{CONCLUSION}

In summary, we have studied the AFM wall motion under an anisotropy gradient based on a one-dimensional model. The domain wall velocity depending on the gradient magnitude and intrinsic physical parameters is simulated based on the LLG equation and also calculated theoretically based on the energy dissipation theorem. The domain wall structure is rather robust for a small gradient and moves at a constant velocity, while accelerates for a large gradient due to the enlargement of the domain wall width during its motion. The domain wall mobility is independent of the lattice dimension and wall type $\left(180^{\circ}\right.$ or $\left.90^{\circ}\right)$, while the mobility is enhanced by the DM interaction. Moreover, the physical mechanism of the AFM wall dynamics being faster than FM wall dynamics is also qualitatively explained. This work unveils a promising strategy for controlling the AFM domain walls, benefiting future spintronic applications.

\section{ACKNOWLEDGMENTS}

We sincerely appreciate the insightful discussions with Zhengren Yan and Huaiyang Yuan. The work is supported by the Natural Science Foundation of China (Grants No. 51971096 and No. 51721001), and the Science and Technology Planning Project of Guangzhou in China (Grant No. 201904010019), and the Natural Science Foundation of Guangdong Province (Grants No. 2019A1515011028 and No. 2016A030308019).

\section{APPENDIX: THE DERIVATION OF THE AFM WALL VELOCITY}

Following earlier works, we use a domain wall ansatz $\tan (\theta / 2)=e^{(z-q) / \lambda}$ for the $180^{\circ}$ wall and $\tan (\theta)=e^{(z-q) / \lambda}$ for the $90^{\circ}$ wall with $q$ being the coordination of the wall center. The wall spins are restricted in the $x-z$ plane $(\varphi=0)$, and the local staggered magnetization is given by

$$
\begin{aligned}
& n_{x}=\sin \left(2 \arctan \left(e^{(z-q) / \lambda}\right)\right) \cos (\varphi), \\
& n_{y}=\sin \left(2 \arctan \left(e^{(z-q) / \lambda}\right)\right) \sin (\varphi), \\
& n_{z}=\cos \left(2 \arctan \left(e^{(z-q) / \lambda}\right)\right)
\end{aligned}
$$

for a $180^{\circ}$ wall, and

$$
\begin{aligned}
& n_{x}=\sin \left(\arctan \left(e^{(z-q) / \lambda}\right)\right) \cos (\varphi), \\
& n_{y}=\sin \left(\arctan \left(e^{(z-q) / \lambda}\right)\right) \sin (\varphi), \\
& n_{z}=\cos \left(\arctan \left(e^{(z-q) / \lambda}\right)\right)
\end{aligned}
$$

for a $90^{\circ}$ domain wall.

According to the classical mechanics, one obtains $d E_{\mathrm{DW}} / d t=-2 R$. The domain wall energy is calculated by 
$E_{\mathrm{DW}}=2(2 J K)^{1 / 2}$ for a $180^{\circ}$ wall and $E_{\mathrm{DW}}=(J K / 2)^{1 / 2}$ for a $90^{\circ}$ wall with $K=K_{0}+v t \Delta K$. Subsequently, $d E_{\mathrm{DW}} / d t$ reads

$$
d E_{\mathrm{DW}} / d t=\left\{\begin{array}{ll}
2 \lambda v \Delta K & \text { for } 180^{\circ} \mathrm{DW} \\
\lambda v \Delta K / 2 & \text { for } 90^{\circ} \mathrm{DW}
\end{array} .\right.
$$

Furthermore, using the mathematical transformation

$$
\dot{\mathbf{n}}=\frac{d \mathbf{n}}{d t}=\frac{d \mathbf{n}}{d z} \frac{d z}{d t}=\frac{d \mathbf{n}}{d z} v,
$$

$R$ can be described by

$$
R=\left\{\begin{array}{ll}
\alpha \rho v^{2} / \lambda & \text { for } 180^{\circ} \mathrm{DW} \\
\alpha \rho v^{2} / 4 \lambda & \text { for } 90^{\circ} \mathrm{DW}
\end{array} .\right.
$$

At last, the domain wall velocity is obtained by

$$
v=-\frac{\gamma \lambda^{2} \Delta K}{\alpha \mu_{s}}
$$

both for $180^{\circ}$ and $90^{\circ}$ walls.
[1] R. Cheng, J. Xiao, Q. Niu, and A. Brataas, Phys. Rev. Lett. 113, 057601 (2014).

[2] E. Gomonay and V. Loktev, Low. Temp. Phys. 40, 17 (2014).

[3] V. Baltz, A. Manchon, M. Tsoi, T. Moriyama, T. Ono, and Y. Tserkovnyak, Rev. Mod. Phys. 90, 015005 (2018).

[4] O. Gomonay, T. Jungwirth, and J. Sinova, Phys. Status Solidi RRL 11, 1770319 (2017).

[5] C. Marrows, Science 351, 558 (2016).

[6] Z. Y. Chen, Z. R. Yan, M. H. Qin, and J. M. Liu, Phys. Rev. B 99, 214436 (2019).

[7] Z. Y. Chen, M. H. Qin, and J. M. Liu, Phys. Rev. B 100, 020402(R) (2019).

[8] T. Kampfrath, A. Sell, G. Klatt, A. Pashkin, S. Mährlein, T. Dekorsy, M. Wolf, M. Fiebig, A. Leitenstorfer, and R. Huber, Nat. Photonics 5, 31 (2011).

[9] W. C. Yu, J. Lan, and J. Xiao, Phys. Rev. B 98, 144422 (2018).

[10] O. Gomonay, T. Jungwirth, and J. Sinova, Phys. Rev. Lett. 117, 017202 (2016).

[11] P. Wadley, B. Howells, J. Železný, C. Andrews, V. Hills, R. P. Campion, V. Novák, K. Olejník, F. Maccherozzi, and S. Dhesi, Science 351, 587 (2016).

[12] Y. L. Zhang, Z. Y. Chen, Z. R. Yan, D. Y. Chen, Z. Fan, and M. H. Qin, Appl. Phys. Lett. 113, 112403 (2018).

[13] S. K. Kim, O. Tchernyshyov, and Y. Tserkovnyak, Phys. Rev. B 92, 020402(R) (2015).

[14] S. Selzer, U. Atxitia, U. Ritzmann, D. Hinzke, and U. Nowak, Phys. Rev. Lett. 117, 107201 (2016).

[15] Z. R. Yan, Z. Y. Chen, M. H. Qin, X. B. Lu, X. S. Gao, and J. M. Liu, Phys. Rev. B 97, 054308 (2018).

[16] K. Olejník, T. Seifert, Z. Kašpar, V. Novák, P. Wadley, R. P. Campion, M. Baumgartner, P. Gambardella, P. Němec, and J. Wunderlich, Sci. Adv. 4, eaar3566 (2018).

[17] S. Y. Bodnar, L. Šmejkal, I. Turek, T. Jungwirth, O. Gomonay, J. Sinova, A. Sapozhnik, H.-J. Elmers, M. Kläui, and M. Jourdan, Nat. Commun. 9, 348 (2018).

[18] X. R. Wang, P. Yan, J. Lu, and C. He, Ann. Phys. (New York) 324, 1815 (2009).

[19] A. Mougin, M. Cormier, J. P. Adam, P. J. Metaxas, and J. Ferré, Europhys. Lett. 78, 57007 (2007).

[20] T. Nozaki, T. Yamamoto, S. Tamaru, H. Kubota, A. Fukushima, Y. Suzuki, and S. Yuasa, APL Mater. 6, 026101 (2018).

[21] S. Z. Peng, S. Li, W. Kang, J. Q. Zhou, N. Lei, Y. G. Zhang, H. X. Yang, X. Li, P. K. Amiri, and K. L. Wang, Appl. Phys. Lett. 111, 152403 (2017).
[22] X. Li, K. Fitzell, D. Wu, C. T. Karaba, A. Buditama, G. Yu, K. L. Wong, N. Altieri, C. Grezes, and N. Kioussis, Appl. Phys. Lett. 110, 052401 (2017).

[23] Z. Wen, H. Sukegawa, T. Seki, T. Kubota, K. Takanashi, and S. Mitani, Sci. Rep. 7, 45026 (2017).

[24] C. Ma, X. Zhang, J. Xia, M. Ezawa, W. Jiang, T. Ono, S. Piramanayagam, A. Morisako, Y. Zhou, and X. Liu, Nano Lett. 19, 353 (2018).

[25] X. Wang, W. Gan, J. Martinez, F. Tan, M. Jalil, and W. Lew, Nanoscale 10, 733 (2018).

[26] H. Y. Xia, C. K. Song, C. D. Jin, J. S. Wang, J. B. Wang, and Q. F. Liu, J. Magn. Magn. Mater. 458, 57 (2018).

[27] L. C. Shen, J. Xia, G. P. Zhao, X. C. Zhang, M. Ezawa, O. A. Tretiakov, X. X. Liu, and Y. Zhou, Phys. Rev. B 98, 134448 (2018).

[28] F. D. M. Haldane, Phys. Rev. Lett. 50, 1153 (1983).

[29] D. Hinzke and U. Nowak, Phys. Rev. Lett. 107, 027205 (2011).

[30] P. Yan, X. S. Wang, and X. R. Wang, Phys. Rev. Lett. 107, 177207 (2011).

[31] E. G. Tveten, A. Qaiumzadeh, and A. Brataas, Phys. Rev. Lett. 112, 147204 (2014).

[32] Y. G. Semenov, X.-L. Li, and K. W. Kim, Phys. Rev. B 95, 014434 (2017).

[33] K. M. Pan, L. D. Xing, H. Y. Yuan, and W. W. Wang, Phys. Rev. B 97, 184418 (2018).

[34] S. K. Kim, Y. Tserkovnyak, and O. Tchernyshyov, Phys. Rev. B 90, 104406 (2014).

[35] P. Wadley, S. Reimers, M. J. Grzybowski, C. Andrews, M. Wang, J. S. Chauhan, B. L. Gallagher, R. P. Campion, K. W. Edmonds, and S. S. Dhesi, Nat. Nanotechnol. 13, 362 (2018).

[36] H. H. Yang, H. Y. Yuan, M. Yan, H. W. Zhang, and P. Yan, Phys. Rev. B 100, 024407 (2019).

[37] K.-S. Ryu, L. Thomas, S.-H. Yang, and S. Parkin, Nat. Nanotechnol. 8, 527 (2013).

[38] S. Emori, U. Bauer, S.-M. Ahn, E. Martinez, and G. S. Beach, Nat. Mater. 12, 611 (2013).

[39] S. G. Je, D. H. Kim, S. C. Yoo, B. C. Min, K. J. Lee, and S. B. Choe, Phys. Rev. B 88, 214401 (2013).

[40] D. H. Kim, D. Y. Kim, S. C. Yoo, B. C. Min, and S. B. Choe, Phys. Rev. B 99, 134401 (2019).

[41] A. Qaiumzadeh, L. A. Kristiansen, and A. Brataas, Phys. Rev. B 97, 020402(R) (2018).

[42] Y. Yamane, Phys. Rev. B 98, 174434 (2018).

[43] Y. J. Zhang, L. Wu, J. Ma, Q. H. Zhang, A. Fujimori, J. Ma, Y. H. Lin, C. W. Nan, and N. X. Sun, npj Quant Mater. 2, 17 (2017). 